\title{
A three-phase analysis of the prevention of recreational softball injuries*
}

\author{
DAVID H. JANDA, † MD, EDWARD M. WOJTYS, $\$$ MD, FRED M. HANKIN, $\|$ MD, \\ MILBRY E. BENEDICT, ${ }^{a}$ MA, AND ROBERT N. HENSINGER,§ MD
}

\begin{abstract}
From † Orthopaedic Surgery Associates, St. Joseph Mercy Hospital, the $\S$ Section of Orthopaedic Surgery, and the a Department of Recreational Sports, University of Michigan, Ann Arbor, Michigan, and || Community Orthopedic Surgery, PC, and Huron Valley Hand Surgery, Ypsilanti, Michigan
\end{abstract}

\begin{abstract}
Recreational sports injuries are expensive to society. Prevention of such injuries must be a major public health goal.

In a previous retrospective study, base sliding was found to be responsible for $71 \%$ of recreational softball injuries. Because most injuries occurred during rapid deceleration against stationary bases, quick-release (break-away) bases were evaluated as a means to modify this mechanism of injury. In a prospective study, 633 softball games were played on break-away base fields and 627 games were played on stationary base fields. Forty-five sliding injuries occurred on the stationary base diamonds (1 injury for every 13.9 games) and only two sliding injuries occurred on the break-away fields ( 1 injury for every 316.5 games). The medical costs for injuries on the stationary base fields was 79 times greater than that on the break-away fields. In a 1035 game follow-up study performed on all fields equipped with break-away bases, two sliding injuries occurred ( 1 injury for every 517.5 games).

Installing break-away bases in fields used by recreational leagues would achieve a significant reduction of serious softball injuries (98\%) and, therefore, should be mandatory.

Based on our findings, the Centers for Disease Control has estimated 1.7 million injuries would be prevented nationally per year, saving $\$ 2.0$ billion per year nationally in acute medical care costs.
\end{abstract}

Injury prevention is a major public health issue. The work-

\footnotetext{
*Presented at the 15th annual meeting of the AOSSM, Traverse City Michigan, July 1989.

$\ddagger$ Address correspondence and reprint requests to: David $H$. Janda, $M D$ Orthopaedic Surgery Associates, P.O. Box 955, Ann Arbor, MI 48106.
}

place, consumer products, and public services are all governed by regulations designed to protect the individual from unnecessary risks. Athletes are also governed by rules and equipment regulations designed to prevent injuries during competition.

An athlete's risk of sustaining a sports-related injury is influenced by several factors. High impact sports, such as football, place the athlete at an increased risk of musculoskeletal trauma. Governing rules that regulate the games can modify injury patterns. Restricting pitching time in Little League baseball is one such example of a healthrelated regulation. Strict enforcement of rules by vigilant officials can foster the safety of the players and greatly reduce the threat of injury. Peterson's 1970 analysis $^{5}$ of injury rates in football led to the elimination of cross-body blocking and hence a substantial reduction in football injuries. Unfortunately, many injuries that predispose the recreational athlete to injury are difficult to control. Poor technique (i.e., sliding), inadequate coaching, lack of conditioning, or concomitant use of alcohol may all contribute to the problem.

It is difficult to control and regulate adherence to safety rules in recreational athletics, even when games are officiated. As a consequence, passive preventive measures that do not depend on the athlete, referee, level of competition, or skill should be incorporated into the sport.

Despite driver education programs, improved road markings, and better road construction, motor vehicle accidents continue to occur. The use of seat belts is an appropriate semipassive preventive measure. However, seat belt use is dependent upon the driver fastening the belt. Recently, a pure passive preventive system has been developed in which the seat belt automatically locks around the driver. Another example of a passive system is the recent institution of airbags in cars. The use of seat belts or airbags does not change any of the underlying causative effects of the acci- 
dent. They do, however, alter the severity of injury to the driver. Examples of similar extrinsic or passive preventive measures in sports include using protective eye wear during racquet sports, head gear during batting, and break-away hockey nets during hockey.

The National Electronic Surveillance Systems of the United States Consumer Product Safety Commission has estimated that softball is the number one sport leading to emergency room visits in the United States. ${ }^{6}$ Further, the American Softball Association has estimated that 40 million individuals, nationally, participate in organized softball leagues, playing an estimated 23 million games per year. In addition to a wide variation in age in these leagues, there is also a wide range in physical condition and athletic ability.

The price of recreational sports injuries can be categorized into short-term and long-term expenditures. Short-term impacts include: acute medical care costs, time lost from work, and expenses related to the injured player's employer concerning replacement or lost production. The long-term consequences include: medical care expenditures, restriction of future athletic activities, permanent functional impairment, and escalating insurance premiums for the injured player, his employer, the field owner, and the softball league itself. The potential costs of these injuries can be staggering; therefore, prevention is of utmost importance.

The consequences of a sports-related injury may include lost wages, restriction of future athletic activities, and longterm functional impairment. In a previous retrospective study, ${ }^{2}$ we identified base sliding as the mechanism responsible for $71 \%$ of recreational softball injuries. This review indicated that a variety of injuries resulted from base sliding, including ligament disruptions and fractures. The most common mechanism of sliding injuries was impact loading of a poorly positioned extremity or joint.

The unexpected costs of these traumatic events were investigated in order to facilitate and emphasize the discussion of their prevention. ${ }^{2}$ The cost of injury to the player, his or her employer, and the sponsoring softball organization can be significant. For instance, athletic knee injuries are common and can post high morbidity for participants. Time lost from work and future functional impairment need to be considered. We explored a variety of preventive methods, including the elimination of sliding, improved instruction in sliding techniques, the use of recessed bases, and the use of break-away bases.

Softball and baseball have, are, and will always be heavily steeped in tradition. We found that making sliding illegal was impractical since giving up sliding was unacceptable to the participants. Instructional clinics were offered; however, few of the recreational athletes attended. Recessed bases, like home plate, are a viable alternative; however, poor visualization caused umpires to object as "safe versus out" calls became an overwhelming problem. These solutions did not deal with the problem of indecision in the mind of the baserunner, poor musculoskeletal conditioning, occasional alcohol consumption, and a desire to impress one's teammates and fans.
With these factors in mind, we felt that an altered base design would provide a practical, reliable, and cost-effective means of reducing sliding injuries. Because most injuries occurred during rapid deceleration against stationary bases, quick-release bases were chosen to modify this mechanism of injury.

\section{METHODS}

Break-away bases were placed on all softball fields at our institutions. The break-away base system used was the Rogers Break-Away base (Elizabethtown, PA). Each set of three bases costs $\$ 350.00$, which is less than twice the cost of a set of standard stationary bases. The system consists of a rubber mat that is set flush with the infield surface and is anchored into the ground by a buried metal post similar to that used with standard stationary bases. Rubber grommets arising from the rubber mat attach to anchoring sockets on the undersurface of the break-away portion of the base. This particular system is available in four models: youth, teen, adult, and professional, each differing in the amount of force needed to cause the base to break away. The difference between the models is in the consistency of the top portion of the base (i.e., the youth model is less rigidly held and breaks away easier than the teen, adult, or professional models. The adult model, which we used, requires 700 foot pounds of force for the break-away portion of the base to release, or $20 \%$ of the force necessary for a stationary base to disassemble. Base sliding injuries that occurred on the study fields were documented by field supervisors, and followup was performed by one of the authors. In addition, local hospital emergency rooms, the Student Health Service, and private practice orthopaedic surgeons were requested to keep logs of patients if they were injured on the study fields; these patients were also seen in followup by the authors.

\section{RESULTS}

In the second phase of the study, ${ }^{3} 633$ games were played on break-away base fields and 627 games were played on stationary base diamonds in the Ann Arbor summer league. The players ranged in age from 18 to 55 years and included college students, laborers, executives, and physicians. Teams were assigned to one of four leagues based on skill level and previous playing experience. Women participated in a coed league. Teams were assigned to playing fields on a random and rotating basis. All fields were maintained in the same manner and all experienced the same weather conditions.

During the two seasons studied in Phase II of the study, a total of 45 sliding injuries occurred on the stationary base fields, while only 2 sliding injuries occurred on the breakaway base fields. This difference was statistically significant $(P<0.001)$. Of the 45 injuries sustained by players sliding into stationary bases, 43 involved the lead foot or hand. The distribution of ankle injuries sustained on the stationary base fields is shown in Table 1. Ankle injuries predominated, accounting for 24 of the 45 total injuries. The total medical 
TABLE 1

Sliding injuries, 1986 to $1987^{3}$

\begin{tabular}{lcc}
\hline \multicolumn{1}{c}{ Type of injury } & $\begin{array}{c}\text { No. of injuries } \\
\text { involving } \\
\text { stationary bases }\end{array}$ & $\begin{array}{c}\text { No. of injuries } \\
\text { involving break- } \\
\text { away bases }\end{array}$ \\
\hline Ankle sprains & 18 & 1 \\
Ankle fractures & 6 & 1 \\
Skin abrasions & 5 & \\
Knee & 3 & \\
$\quad$ MCL sprain & 2 & \\
ACL tear & 1 & \\
Tibia/fibula fractures & 1 & \\
Shoulder subluxation & 1 & \\
AC Joint Injury (Type I) & 1 & \\
Wrist fractures & 1 & \\
Wrist sprains & 1 & \\
Foot contusion/sprain & 1 & \\
Finger ligament injury (volar plate) & 1 & \\
Finger dislocation & 3 & \\
Finger fracture & 45 & \\
Totals & & \\
\hline
\end{tabular}

charges for these 45 players was approximately $\$ 55,050$ (\$1223 per injury). Two other players were injured when they tripped over the stationary bases as they ran around the diamond. One of these players sustained an ankle fracture and the other a scaphoid fracture. However, these injuries were not included in our analysis since they did not occur as a result of sliding, and we were unsure of whether break-away bases could have prevented them.

Two isolated injuries occurred on the break-away bases: a nondisplaced medial malleolar ankle fracture and an ankle sprain. The total medical charges for these two players was approximately $\$ 700.00$ ( $\$ 350.00$ per injury). It should be noted that in these two sliding injuries, the bases did not break away. Opposing players were not standing on the bases during these occurrences.

The director of field supervisors was interviewed two or three times each month during the study concerning experiences with the break-away bases. The field supervisors felt that softball play was not significantly delayed with the use of break-away bases, even though sliding players broke away the bases up to six times during each game. Properly seated break-away bases did not detach during routine base running. The umpires did not have difficulty with judgment calls (safe versus out) when the bases released. For continuation of play circumstances, when the break-away portion of the base did break away, the rubber mat that is flush with the infield surface was considered the base when determining if the runner was safe or out.

Finally, in Phase III of the study, we performed a longterm followup of 1035 games played on fields that had all been switched over to break-away bases. The same surveillance system was used. Two injuries occurred during the study period. Each player sustained an ankle sprain that was treated with protected weightbearing. The total medical cost for these injuries was $\$ 400.00$.

One player sustained a lateral collateral ligament ankle sprain to his left foot, which was not his lead leg. This injury was sustained as the patient caught his cleat on the groundhe never reached the base. The second injured player sus- tained a deltoid ligament sprain to her lead foot while sliding into the base.

\section{DISCUSSION}

Having identified sliding as the mechanism most frequently leading to softball injuries, we instituted a prospective study addressing this mechanism of injury. A follow-up study then reinforced our initial findings. The prospective study supports the concept that modifying the bases can alter the frequency of sliding injuries. An analysis of our injury rates reveals that one injury occurred in every 13.9 games $(7.2 \%)$ on the stationary base fields, while on the break-away base fields, one injury occurred every 316.5 games $(0.3 \%)$. So, for each sliding injury on the break-away base field, there were 22.7 injuries on the stationary base field. Even with breakaway bases, injuries will still occur. Most will be a result of judgment errors by the runner. Improper sliding technique, poor timing, inadequate physical conditioning, and alcohol consumption contribute to sliding injuries. Break-away bases, however, can modify the outcome when these factors are involved.

In our prospective followup of 1035 consecutive games with break-away bases, two sliding injuries occurred. When comparing these results to our results in Phase II of the study, the trend of diminishing sliding injuries secondary to break-away bases was reinforced. In Phase III, one injury occurred for every 517.5 games $(0.19 \%)$.

In a biomechanical study of sliding by Corzatt et al., ${ }^{1}$ sliding was analyzed kinematically. Four phases were identified: the sprint, attainment of the sliding position, the airborne phase, and the landing phase. The authors indicate that injuries occurred in the last phase, the landing phase, where a small amount of the body area is not only used to absorb the shock of impact but also is subjected to high horizontal velocities as the base is contacted.

The quick-release feature of the break-away bases decreases the impact load generated against the athlete's limb and the subsequent trauma inflicted upon it. Sliding players come in all sizes and approach the bases from all angles, so that no one preventive system can be completely foolproof. The forces generated by the trajectory athlete against the ground or other players may still be more than sufficient to result in severe injuries to the musculoskeletal system.

Prevention of sliding injuries would be beneficial to the athlete. Reduction of losses sustained by the player, the player's employer, and insurance carriers could be realized. The extra cost for a set of break-away bases ( $\$ 350$ compared to $\$ 180$ a set for standard bases) is far outweighed by the potential savings in health care costs if a sliding injury can be prevented.

An actuarial analysis by the Section of Epidemiology and Injury at the Centers for Disease Control has estimated, based on Phase II of our study, that by changing from stationary to break-away bases across the United States, approximately 1.7 million injuries per year would be prevented and over $\$ 2.0$ billion in medical care costs per year could be saved. ${ }^{4}$ 
Injuries are inherent in any recreational activity. Most base sliding accidents result from judgment errors of the runner, poor sliding technique, and inadequate physical conditioning. Break-away bases can serve as a passive intervention to modify the outcome of these factors. The use of break-away bases decreases injuries without player involvement or altering the play, excitement, entertainment, competition or interest in the game. This intervention was also independent of players, umpires, weather, or the time of day.

Sports-related injuries are expensive for the player, the employer, and the insurance carrier. Economic costs are, of course, an important concern when the long-term health of the athlete is impaired by a sliding injury. The use of breakaway bases decreases the number and severity of softball injuries. Break-away bases are cost-effective and safer than standard stationary bases. In recreational softball leagues, the use of break-away bases should be mandatory.

\section{ACKNOWLEDGMENTS}

The authors gratefully acknowledge the invaluable help of Dr. David Amato of the Department of Biostatistics, Uni- versity of Michigan, for his help with the statistical analysis, and the Rogers Sports Corporation based in Elizabethtown, Pennsylvania, for the donation of some of the bases used in this study.

\section{REFERENCES}

1. Corzatt RD, Groppel JL, Pfaustsch $E$, et al: The biomechanics of headfirst versus feet-first sliding. Am J Sports Med 12: 229-232, 1984

2. Janda DH, Hankin FM, Wojtys EM: Softball injuries, cost, cause, prevention. Am Fam Physician 33: 143-144, 1986

3. Janda DH, Wojtys EM, Hankin FM, et al: Softball sliding injuries. A prospective study comparing standard and modified bases. JAMA 259: $1848-1850,1988$

4. Janda $\mathrm{DH}$, Wojtys EM, Hankin FM, et al: Softball sliding injuries-Michigan, 1986-1987. Morbidity and Mortality Weekly Report 37(11): 169-170, 1988

5. Peterson TR: The cross-body block, the major cause of knee injuries. JAMA 211: 211-214, 1970

6. Product Summary Report: National Electronic Injury Surveillance System/ Hazard Identification and Analysis. National Injury Information Clearinghouse, June 1982, pp 19-20 